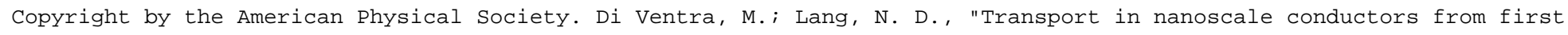
principles," Phys. Rev. B 65, 045402 DOI: http://dx.doi.org/10.1103/PhysRevB.65.045402

PHYSICAL REVIEW B, VOLUME 65, 045402

\title{
Transport in nanoscale conductors from first principles
}

\author{
Massimiliano Di Ventra ${ }^{1}$ and Norton D. Lang ${ }^{2}$ \\ ${ }^{1}$ Department of Physics and Center for Self-Assembled Nanostructures and Devices, \\ Virginia Polytechnic Institute and State University, Blacksburg, Virginia 24061 \\ ${ }^{2}$ IBM Research Division, Thomas J. Watson Research Center, Yorktown Heights, New York 10598
}

(Received 22 August 2001; published 14 December 2001)

\begin{abstract}
We describe a first-principles atomistic approach to calculate the electronic and atomic dynamics of nanoscale conductors under steady-state current flow. The approach is based on a self-consistent solution of the Lippmann-Schwinger equation within the density-functional formalism for a sample connected to two bare metallic electrodes with a finite bias. Three-terminal device geometries can also be described easily using the present approach. The formalism provides the most fundamental quantities to describe the dynamics of the whole system: the self-consistent electronic wave functions. With these, the forces on the atoms are determined according to a Helmann-Feynman-like theorem that takes into account the contribution of the continuum of states as well as of the discrete part of the spectrum. Examples of applications will be given in the case of molecular devices with different anchoring groups at the interface between the molecule and the electrodes. In particular, we find that conductances close to the quantum unit $\left(2 e^{2} / h\right)$ can be achieved with a given molecular structure simply by increasing the atomic number of the anchoring group.,

DOI: 10.1103/PhysRevB.65.045402

PACS number(s): 73.40.Jn, 73.40.Cg, 73.40.Gk, 85.65.+h
\end{abstract}

\section{INTRODUCTION}

In the area of molecular electronics, ${ }^{1}$ it is very important to be able to calculate the properties of basic circuit elements from first principles. $^{2-9}$ Because of the difficulty of doing well-characterized experiments on such individual elements, theoretical calculations play a significant role in guiding experiments, as well as in understanding their results. However this is only fully possible, particularly for the case in which the experiment has not yet been done, if there are no adjustable or unknown parameters in the calculation.

The transport properties of atomic-scale wires ${ }^{10}$ and of relatively small molecules ${ }^{11}$ (which may function as wires or as switching devices) connecting more extended electrodes are strongly affected by the relative positions of the Fermi levels of the electrodes and the energy levels of the molecular circuit elements, and by the broadening of these molecular levels. ${ }^{2-5}$ The relative level positioning affects, and is in turn affected by, the charge transfer between the electrodes and the circuit elements; and this charge transfer itself has an important effect on the transport properties. Determining these level positions and charge transfers correctly requires that the problem be solved fully self-consistently: small errors in the charge transfer can lead to substantial errors in the level positions, which can result in an incorrect account of the transport. Self-consistency is also required to determine accurately the current-induced forces on the atoms that comprise the circuit elements, so as to account for such effects as electromigration.

The method we describe here uses the density-functional formalism ${ }^{12}$ to calculate the wave functions, electron-density distributions, and potentials of the systems of interest. With wave functions in particular, the other steady-state properties of interest, like, e.g., current-induced forces, can be determined. We use the Lippmann-Schwinger equation to express each individual continuum wave function of the full molecule-electrode system in terms of the corresponding wave function of the biased electrodes without the molecule (or atomic wire), and the difference in the total effective potential between the electrode-molecule system and the electrodes alone. This potential difference vanishes away from the region of the molecule, and so the self-consistent part of the calculation can be confined to this spatial region.

As an example of an application of the method, we study the conductance of a molecular device with different anchoring groups at the interface between the wire and the electrodes, in particular S, Se, and Te. We find that the conductance increases with increasing atomic number, and assumes a value close to the quantum of conductance $\left(2 e^{2} / h\right)$ in the case of Te. This study thus confirms, once again, the importance of the contacts in determining the absolute value of the current in molecular wires, and suggests that contact engineering can eventually lead to practical applications of molecules as electronic components.

\section{CALCULATION OF THE CURRENT}

The physical system of interest to us consists of a sample (e.g., a molecule, or any set of atoms) between two bulk electrodes which are kept at a certain external potentialenergy difference $V_{B}=E_{F R}-E_{F L}$ between the right $\left(E_{F R}\right)$ and left $\left(E_{F L}\right)$ Fermi levels (see Fig. 1, right panel). The
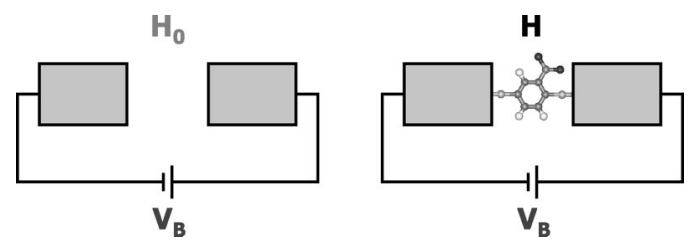

FIG. 1. Left panel: schematic of two bare electrodes kept at a certain external potential difference. The biased bare electrodes are represented by the unperturbed Hamiltonian $H_{0}$. Right panel: schematic of the total system (sample plus electrodes). The sample is, e.g., a molecule, or any set of atoms. 


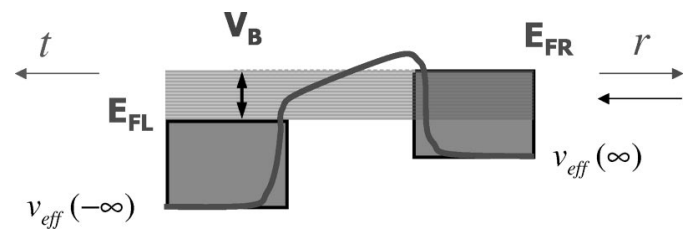

FIG. 2. Schematic of the two bare electrodes kept at a certain external potential difference, and the corresponding effective potential $v_{\text {eff }}$. The left electrode is positively biased: electrons incident from the right electrode are partly transmitted into the left electrode with a probability $t$, and reflected back into the right electrode with a probability $r$.

sample is part of an external circuit, which is assumed to have zero impedance. We consider the case of a steady-state (dc) current flow. Current and voltage fluctuations are assumed to be zero. The study of these fluctuations will be the subject of a forthcoming publication. ${ }^{13}$ We also focus our attention on those systems where strong correlation effects are negligible. Many physical systems presently investigated fall in this category. ${ }^{11}$ Since the electron mean free path in nanoscale conductors is much longer than the contact constriction length, polaronic effects are also expected to be negligible for short sample lengths - the ones considered in the present paper. ${ }^{14,15}$ In the following we will discuss the case of transport at zero temperature only. Inclusion of the FermiDirac distribution function in the calculation of the current is straightforward.

For clarity of discussion, in the following we shall assume that the left-hand electrode is positively biased. Electrons thus travel from the right electrode without scattering until they reach a region in space close to the sample region. The system Hamiltonian can be written as $H=H_{0}+V$, where $H_{0}$ describes the energy spectrum of the two bulk electrodes kept at a finite bias, without the sample atoms in between. $V$ is the perturbation potential upon which electrons, in stationary states of the electrodes, scatter elastically. The Hamiltonian $H$ will be described within the density-functional theory of many-electron systems. ${ }^{12}$

\section{A. Bare electrodes}

In Fig. 2 we represent schematically the total effective potential $v_{\text {eff }}(z)$ (sum of the electrostatic and exchangecorrelation potentials) for the two bare electrodes at a given external bias, where $z$ is the coordinate perpendicular to the electrode surfaces. Assuming that equilibrium has been reached (steady-state condition), the potential $v_{\text {eff }}(z)$ tends toward a constant value $v_{\text {eff }}(-\infty)$ deep in the left-hand electrode, and to a constant value $v_{\text {eff }}(\infty)$ deep in the right-hand electrode (see Fig. 2). The stationary states of $H_{0}$ will have different asymptotic forms, according to their energy. In the energy region between $v_{\text {eff }}(-\infty)$ and $v_{\text {eff }}(\infty)$, the wave functions of $H_{0}$ are phase-shifted sine waves deep in the left electrode, which decay exponentially toward the right. In the energy region between $v_{\text {eff }}(\infty)$ and $E_{F L}$, the stationary states have two different asymptotic forms: (1) a plane wave incident on the barrier region from the left together with its reflected and transmitted parts, and (2) a plane wave incident on the barrier region from the right together with its reflected and transmitted parts. ${ }^{16}$ Finally, for energies $E_{F L} \leqslant E$ $\leqslant E_{F R}$, the wave functions of interest correspond to waves incident from the right electrode (plus their reflected and transmitted parts). As we will discuss in Sec. II B, the potential $V$ tends asymptotically to zero more rapidly than $1 / r$. In this case, the stationary states of $H_{0}$ (and, therefore, the stationary states of $H$ ) with a given energy $E_{F L} \leqslant E \leqslant E_{F R}$, momentum $\mathbf{K}_{\|}$, and $\operatorname{spin}^{17}$

$$
\Psi_{E \mathbf{K}_{\|}}^{0}(\mathbf{r})=e^{i \mathbf{K}_{\|} \cdot \mathbf{R}_{u_{E \mathbf{K}_{\|}}}(z),}
$$

satisfy the scattering boundary conditions (in atomic units) ${ }^{18}$

$$
u_{E \mathbf{K}_{\|}}(z)=(2 \pi)^{3 / 2} k_{R}^{-1 / 2} \times \begin{cases}e^{-i k_{R} z}+R e^{i k_{R} z}, & z \rightarrow \infty \\ T e^{-i k_{L} z}, & z \rightarrow-\infty\end{cases}
$$

where $\quad \frac{1}{2} k_{R}^{2}=E-\frac{1}{2}\left|\mathbf{K}_{\|}\right|^{2}-v_{\text {eff }}(\infty), \quad \frac{1}{2} k_{L}^{2}=E-\frac{1}{2}\left|\mathbf{K}_{\|}\right|^{2}$ $-v_{\text {eff }}(-\infty)$, and $\mathbf{R}$ is the coordinate parallel to the surfaces. The wave functions $\Psi^{0}$ satisfy the continuum normalization condition

$$
\int d^{3} \mathbf{r}\left[\Psi_{E^{\prime} \mathbf{K}_{\|}^{\prime}}^{0}(\mathbf{r})\right]^{*} \Psi_{E \mathbf{K}_{\|}}^{0}(\mathbf{r})=\delta\left(E^{\prime}-E\right) \delta\left(\mathbf{K}_{\|}^{\prime}-\mathbf{K}_{\|}\right) .
$$

Since the details of the electrodes are not important up to the interface with the sample, we represent them using a uniform-background (jellium) model. ${ }^{5}$ The jellium model also allows an easy enumeration of scattering states, and avoids complications arising from band crossing in the electronic structure of the electrodes. Layers of atoms at the interface, and the sample atoms are represented within a pseudopotential framework, and constitute the scattering potential $V$ (see below). In previous work ${ }^{2}$ we showed that, in the case of gold electrodes, the jellium model correctly represents the scattering properties of molecular structures making contact with a flat surface. For other types of substrates the use of jellium is not a limitation either: the extra resistance between the jellium and the interface atoms, if any, can be easily determined, since it makes up for the difference between the current with and without the extra interface layers.

The calculation of the electronic density and potential for the bare electrodes in the presence of the external bias is done within density-functional theory in the local-density approximation. $^{12}$ The calculation starts with a potential $v_{\text {eff }}(z)$ equal to the band bottom $v_{\text {eff }}(-\infty)$ deep in the left electrode, and equal to $V_{B}+v_{\text {eff }}(\infty)$ deep in the right electrode. However, an electron current flows from the right electrode to the left electrode, leading to a slightly larger density of electrons in the left electrode and, consequently, to a deficit of electron density in the right electrode. These changes are present within a mean free path length of the surfaces. Since charge neutrality has to be satisfied deep within both electrodes, the Fermi level relative to the bottom of the band, in each of the two electrodes, has to change self-consistently from its free-electron value. Therefore, $E_{F L}-v_{\text {eff }}(-\infty)$ decreases and $E_{F R}-v_{\text {eff }}(\infty)$ increases. Since $V_{B}=E_{F R}-E_{F L}$, 
then $v_{\text {eff }}(\infty)-v_{\text {eff }}(-\infty)<V_{B}$. Even if this effect is negligible for large electrode spacing, it is important, and needs to be taken into account in the other cases.

On each iteration of the self-consistency loop, the computed electron densities deep in each of the two electrodes are renormalized to produce local charge neutrality, and a small charge distribution is added in the region of the surfaces, ${ }^{19}$ that both guarantees overall system neutrality and guarantees that $V_{B}=E_{F R}-E_{F L}$. No such renormalizations or additions of charge are required once self-consistency is achieved, in order to satisfy the neutrality conditions and to give the required separation of the Fermi levels. Finally, with these self-consistent wave functions the Green's function of the bare electrodes $G_{E}^{0}$ is calculated for each energy $E$ in the continuum (see the Appendix).

\section{B. Scattering potential}

The potential $V$ describes the difference in potential between the complete system and the bare electrodes. The choice of what part of the system is described by $H_{0}$ and what is described by $V$ is somewhat arbitrary. However, if no single-electron charging of the sample is assumed, $V$ tends asymptotically to zero more rapidly than $1 / r$. In this case, we can represent $V$ in a box large enough to contain the perturbation of the sample atoms on the bare electrode system. We choose to include in $V$ all sample atoms and interface atoms, if any. All atoms are represented within a pseudopotential approach. ${ }^{20} V$ is therefore

$$
\begin{aligned}
V\left(\mathbf{r}, \mathbf{r}^{\prime}\right)= & v_{p s}\left(\mathbf{r}, \mathbf{r}^{\prime}\right)+\left[v_{x c}[n(\mathbf{r})]-v_{x c}\left[n^{0}(\mathbf{r})\right]\right. \\
& \left.\left.+\int d^{3} \mathbf{r}^{\prime \prime}\left[\delta n \mathbf{r}^{\prime \prime}\right)\right] /\left|\mathbf{r}-\mathbf{r}^{\prime \prime}\right|\right] \delta\left(\mathbf{r}-\mathbf{r}^{\prime}\right)
\end{aligned}
$$

The term $v_{p s}\left(\mathbf{r}, \mathbf{r}^{\prime}\right)$ is the sum of the nonlocal pseudopotentials representing the atomic cores, $v_{x c}[n(\mathbf{r})]$ is the exchange-correlation potential, $n^{0}(\mathbf{r})$ is the electron density for the pair of biased metal electrodes, $n(\mathbf{r})$ is the corresponding quantity for the total system, and $\delta n(\mathbf{r})$ is their difference. The exchange-correlation potential is assumed to be the one for electrons in the ground state at zero bias, and we represent it in the local-density approximation. ${ }^{12}$ This assumption is certainly correct for small external biases, and seems to give quite reasonable results for the transport properties of molecular devices also for larger biases. ${ }^{2}$

\section{Lippmann-Schwinger equation}

Once the Hamiltonian $H_{0}$, of the bare electrodes, and the scattering potential $V$ have been defined, we need to find the self-consistent solutions of the equation $H \Psi_{E}=E \Psi_{E}$. The total current will finally be calculated in terms of these wave functions. For the wave functions in the continuum, this equation can be put exactly into Lippmann-Schwinger form:

$$
\begin{aligned}
& \Psi_{E \mathbf{K}_{\|}}(\mathbf{r}) \\
& \quad=\Psi_{E \mathbf{K}_{\|}}^{0}(\mathbf{r})+\int d^{3} \mathbf{r}^{\prime} d^{3} \mathbf{r}^{\prime \prime} G_{E}^{0}\left(\mathbf{r}, \mathbf{r}^{\prime}\right) V\left(\mathbf{r}^{\prime} \mathbf{r}^{\prime \prime}\right) \Psi_{E \mathbf{K}_{\|}}\left(\mathbf{r}^{\prime \prime}\right) .
\end{aligned}
$$

The wave functions $\Psi_{E \mathbf{K}_{\|}}$also have the same labeling as the wave functions $\Psi_{E \mathbf{K}_{\|}}^{0}$, even though $\mathbf{K}_{\|}$is no longer a good quantum number. The bound states $\Psi_{i}(\mathbf{r})$ of $H$, if any, are calculated by a direct diagonalization of the total Hamiltonian $H$. Only those states that have an energy below $v_{\text {eff }}(-\infty)$ are truly bound states of the total system; these are of course included in the total density. The total density of the system is

$$
n(\mathbf{r})=2 \sum_{i}\left|\Psi_{i}(\mathbf{r})\right|^{2}+2 \int d E \int d^{2} \mathbf{K}_{\|}\left|\Psi_{E \mathbf{K}_{\|}}(\mathbf{r})\right|^{2},
$$

where the factor 2 is for spin degeneracy (we assume the system unpolarized), and the integrals in $E$ and $\mathbf{K}_{\|}$cover the whole continuum spectrum occupied by the electrons.

The self-consistent solution of Eq. (5) starts with a guess of the electronic density, and hence of the potential $V$. The wave functions $\Psi_{E \mathbf{K}_{\|}}(\mathbf{r})$ and $\Psi_{i}(\mathbf{r})$ are then used to compute the new density [Eq. (6)], and the process is iterated until self-consistency is achieved in the density and/or potential $V$.

The total electric current density in the full system is given by

$$
\mathbf{j}(\mathbf{r})=-2 \int_{E_{F L}}^{E_{F R}} d E \int d^{2} \mathbf{K}_{\|} \operatorname{Im}\left\{\left[\Psi_{E \mathbf{K}_{\|}}(\mathbf{r})\right]^{*} \nabla \Psi_{E \mathbf{K}_{\|}}(\mathbf{r})\right\}
$$

where the integral over $\mathbf{K}_{\|}$is restricted to $\left|\mathbf{K}_{\|}\right|$ $\leqslant \sqrt{2\left[E-v_{\text {eff }}(\infty)\right]}$. The total current in the full system is simply the integral over a surface of Eq. (7). However, the current across an infinite surface is infinite in this problem. Therefore, expression (7) is not useful by itself. Since the current of interest to us is the extra current $\delta J$ that flows in the sample with respect to the current for the bare electrodes, we can define $\delta J$ as

$$
\delta J=\int d^{2} \mathbf{R} \hat{z} \cdot\left[\mathbf{j}(\mathbf{r})-\mathbf{j}^{0}\right]
$$

where $\mathbf{j}^{0}$ is the current density for the pair of biased electrodes, and $\hat{z}$ is the unit vector pointing toward the right and perpendicular to the surfaces of the electrodes. Equation (8) provides a finite quantity independent of $z$, and a meaningful definition of the additional current flowing in the sample region. ${ }^{5}$ It is convenient to write the total wave functions as $\Psi=\Psi^{0}+\delta \Psi$ (see below). In this case the additional current can be written as 


$$
\begin{aligned}
\delta J= & -2 \int_{E_{F L}}^{E_{F R}} d E \int d^{2} \mathbf{K}_{\|} \int d^{2} \mathbf{R} \\
& \times \operatorname{Im}\left\{\left[\Psi_{E \mathbf{K}_{\|}}^{0}(\mathbf{r})\right]^{*} \frac{d}{d z} \delta \Psi_{E \mathbf{K}_{\|}}(\mathbf{r})\right. \\
& \left.+\delta \Psi_{E \mathbf{K}_{\|}}^{*}(\mathbf{r}) \frac{d}{d z} \Psi_{E \mathbf{K}_{\|}}^{0}(\mathbf{r})+\delta \Psi_{E \mathbf{K}_{\|}}^{*}(\mathbf{r}) \frac{d}{d z} \delta \Psi_{E \mathbf{K}_{\|}}(\mathbf{r})\right\} .
\end{aligned}
$$

\section{Three-terminal devices}

The above approach can easily be extended to calculate transport in the presence of a third terminal (gate) field. The gate field can be introduced simply as a capacitor field generated by two charged disks at a certain distance from each other. The simplest geometry consists of the sample placed within the capacitor, with the axis of the capacitor perpendicular to the transport direction. ${ }^{4}$

\section{E. Basis set}

We choose plane waves to represent the wave functions. This choice has a number of advantages: (1) quantities like the Green's function and the potential $V$ can be written analytically in this basis set (see the Appendix); (2) convergence can be easily checked by increasing the number of plane waves in the calculation; (3) since the current is mostly derived from wave functions that have a plane-wave character [see Eq. (2)], its convergence is generally fast (5-10 iterations are generally enough to converge the total current); and (4) as we will discuss below, we do not need to evaluate Pulay-like forces ${ }^{21}$ in the calculation of current-induced forces.

All calculations are performed within a box large enough that the potential $V$ is negligible outside the box; therefore, the only question that arises is how good a plane-wave representation is for a finite system. We employ Hockney's method for the Fourier-transform solution of Poisson's equation for isolated systems. ${ }^{22}$ We refer the reader to the original papers for the detailed implementation of the method. We only note here that this method gives an exact representation of the potential inside the box, i.e., the effects of periodicity, intrinsic in the representation, are completely suppressed in the box. ${ }^{22}$ This way, we do not have to deal with any periodic repetition of the potential $V$.

\section{CURRENT-INDUCED FORCES}

We have assumed so far that all atoms in the sample are kept fixed at a given equilibrium position. However, due to the current flow, atom motion can occur in the sample, a phenomenon known as electromigration. ${ }^{23}$ It was recognized in early theoretical work ${ }^{24-27}$ that current-induced forces on a given physical system depend strongly on the microscopic details of the self-consistent electric field that is created upon scattering of the electrons across the region of interest. Selfconsistency in the calculation of the local electric field with the correct scattering boundary conditions is thus essential to have meaningful quantitative results for current-induced forces. The approach outlined above thus provides the necessary ingredients to calculate current-induced forces.

\section{Hellmann-Feynman theorem for transport}

Once a self-consistent solution of the transport problem has been obtained at a given geometry, current-induced relaxation effects are taken into account by calculating forces according to the Hellmann-Feynman-like theorem developed in Ref. 28. The details of the theorem can be found in Ref. 28. Here we outline its main result and practical implementation.

Let us consider the sample and the two electrodes as in Fig. 1. As described above, each of these systems can be treated separately by density-functional theory. Only the potential $V$ of the total Hamiltonian $H=H_{0}+V$ depends on the position of the atoms. The total Hamiltonian of the system can have a discrete and a continuum part. For single-particle wave functions in the discrete part of the spectrum $\Psi_{i}$, square integrability is satisfied. For each energy in the continuum between $v_{\text {eff }}(-\infty)$ and $E_{F R}$ (assuming the left-hand electrode to be positively biased), we build square-integrable wave functions $\Psi_{E \mathbf{K}_{\|} \Delta}$ in an energy region $E<E^{\prime}<E+\Delta$,

$$
\Psi_{E \mathbf{K}_{\|} \Delta}=\mathcal{A} \int_{E}^{E+\Delta} d E^{\prime} \Psi_{E^{\prime} \mathbf{K}_{\|}},
$$

where $\mathcal{A}$ is a normalization constant,

$$
\mathcal{A}=\left(\lim _{\Delta \rightarrow 0} \frac{1}{\Delta}\left\langle\Psi_{E \mathbf{K}_{\|} \Delta} \mid \Psi_{E \mathbf{K}_{\|} \Delta}\right\rangle\right)^{-1 / 2},
$$

and $\Psi_{E}$ 's are the continuum wave-function solutions of the Lippmann-Schwinger equation (5). The force $\mathbf{F}$ acting on a given atom at position $\mathbf{R}_{0}$ due to the electron distribution as modified by the external bias can be shown to be $\mathrm{e}^{28}$

$$
\begin{aligned}
\mathbf{F}= & \sum_{i}\left\langle\Psi_{i}\left|\frac{\partial H}{\partial \mathbf{R}_{0}}\right| \Psi_{i}\right\rangle \\
& +\lim _{\Delta \rightarrow 0} \int_{\sigma} d E \int d^{2} \mathbf{K}_{\|}\left\langle\Psi_{E \mathbf{K}_{\|} \Delta}\left|\frac{\partial H}{\partial \mathbf{R}_{0}}\right| \Psi_{E \mathbf{K}_{\|} \Delta}\right\rangle .
\end{aligned}
$$

The sum and integral in Eq. (12) also include spin variables, $\sigma=\left[v_{\text {eff }}(-\infty), E_{F R}\right]$ is the part of the continuous spectrum occupied by the electrons at a given bias, and $\left|\mathbf{K}_{\|}\right|$ $\leqslant \sqrt{2\left[E-v_{\text {eff }}(-\infty)\right]}$. An additional ion-ion interaction term must be added to the above expression for the total force. Writing the total wave functions $\Psi=\Psi^{0}+\delta \Psi$ as above, the term in Eq. (12) derived only from the bare-electrode wave functions $\Psi^{0}$ can be interpreted as the direct force on the ions. Since this contribution to the total force can be calculated from the electrostatic potential of the bare electrodes, its value is known with essentially arbitrary accuracy. The electron-wind force is also included in Eq. (12) (see Refs. 28 and 24). We note, however, that we did not include inelastic scattering processes in the total Hamiltonian $H$; therefore, no extra modulation of the carrier density in the sample due to dissipation effects is considered in the present case. ${ }^{27}$ 

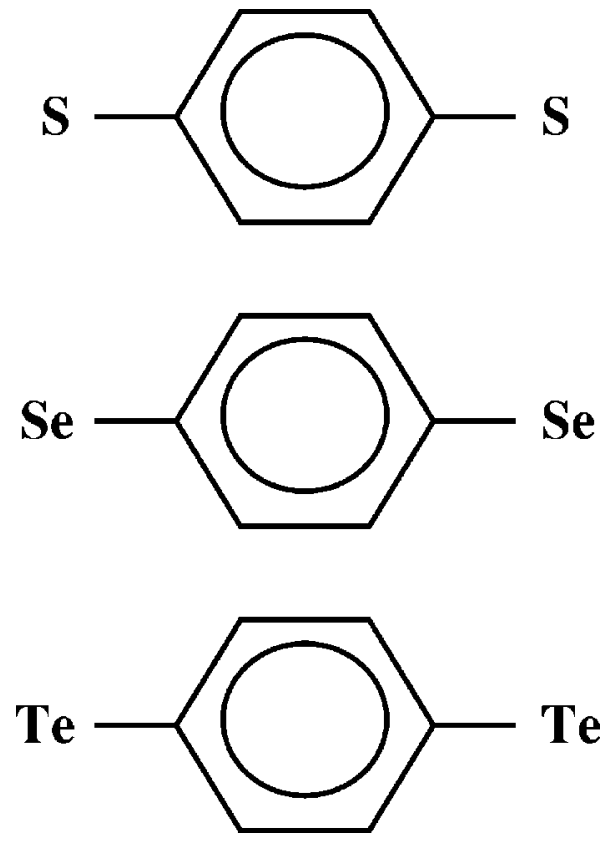

FIG. 3. Schematic of the three molecular structures investigated. The molecules consist of a benzene ring where two opposite $\mathrm{H}$ atoms are replaced by $\mathrm{S}, \mathrm{Se}$, or Te. These anchoring groups make contact to ideal metallic electrodes.

Due to the choice of plane waves as the basis set, Pulaylike forces, ${ }^{21}$ arising from the possible dependence of the basis set on the atomic positions are identically zero and need not be included in Eq. (12). ${ }^{28}$ We divide the energy region $\sigma$ into a discrete set of energy values. Convergence is checked by increasing the number of energy points until the limit in Eq. (12) is accurately described. Also note that, generally, a number of plane waves larger than the one required to converge the current is necessary to obtain converged values for the forces. Starting from a given atomic configuration (e.g., the atoms at the equilibrium experimental atomic positions), we calculate the forces acting on each atom. We then move the atoms according to the gradient of these forces until the force on each atom is zero (generally lower than a defined precision).

\section{SELECTED RESULTS ON MOLECULAR WIRES}

As an example of application, here we consider the conductance of specific molecular wires for a small external bias $V_{B}=0.01 \mathrm{~V}$. In Fig. 3 we plot a schematic of the three molecular wires investigated. The molecules consist of a benzene ring where two opposite $\mathrm{H}$ atoms are replaced by $\mathrm{S}, \mathrm{Se}$, or Te. These anchoring groups make contact to ideal metallic electrodes. The interior electron density of the electrodes is taken to be equal to the value for metallic gold $\left(r_{s} \approx 3\right)$. Over 4000 plane waves were used to converge the current, eight times that number to converge the electron density, and 128 energy points to represent the continuum. In all three cases considered, several molecular states fall below the lowest conduction-band bottom (in the present case, below the conduction-band bottom of the left electrode), and are thus truly bound states.
TABLE I. Conductance $G$ in units of the quantum of conductance $\left(2 e^{2} / h\right)$, density of states at the Fermi level $\operatorname{DOS}\left(E_{F}\right)$, and $G_{0} \times \operatorname{DOS}\left(E_{F}\right) / \operatorname{DOS}\left(E_{\text {peak }}\right)$ of the three molecules of Fig. 3 with different end groups.

\begin{tabular}{lccc}
\hline \hline Group & $\begin{array}{c}G \\
\left(2 e^{2} / h\right)\end{array}$ & $\begin{array}{c}\operatorname{DOS}\left(E_{F}\right) \\
(\text { states/eV })\end{array}$ & $\begin{array}{c}G_{0} \times \operatorname{DOS}\left(E_{F}\right) / \operatorname{DOS}\left(E_{\text {peak }}\right) \\
\left(2 e^{2} / h\right)\end{array}$ \\
\hline $\mathrm{S}$ & 0.036 & 0.09 & 0.051 \\
$\mathrm{Se}$ & 0.12 & 0.64 & 0.15 \\
$\mathrm{Te}$ & 0.88 & 6.96 & 0.90 \\
\hline \hline
\end{tabular}

The atomic configuration of the molecule with $\mathrm{S}$ atoms has been relaxed at zero bias using Eq. (12). ${ }^{29}$ About 6500 plane waves were used to converge the forces. The relaxed configuration consists of C-C bond lengths of $1.40 \AA$, C-H bond lengths of $1.09 \AA, \mathrm{C}-\mathrm{S}$ bonds of $1.70 \AA$, and S-jellium surface bond length of $1.00 \AA$. The latter is in agreement with the equilibrium distance of sulfur adsorption on jellium surfaces, ${ }^{30}$ and the other bond lengths are in good agreement with the experimental bond lengths in isolated benzene molecules and adsorbed thiophenol molecules. ${ }^{31}$ No sizable change in bond lengths is observed for $V_{B}=0.01 \mathrm{~V}$. Since we are interested in determining trends with different anchoring groups, we assume the same bond lengths for the other two molecules where $\mathrm{S}$ is replaced by Se and Te, respectively. We do not expect large changes in the conductance for small variations of Se-, Te-metal distance and Se-,Te-C distance.

Using a tight-binding approach, Yaliraki et al. previously reported $^{32}$ that molecules with $\mathrm{Se}$ anchoring groups show a larger conductance than molecules with $\mathrm{S}$ (by a factor of 25). On the other hand, density-functional calculations without the inclusion of an external bias suggested that $S$ provides the best (in terms of conductance) contact, followed closely by $\mathrm{Se}$ and $\mathrm{Te}{ }^{33}$ In Table I we report the conductance for three molecules in units of the quantum of conductance $\left(G_{0}=2 e^{2} / h\right)$. In the same table we also report the density of states at the Fermi level $\left[\operatorname{DOS}\left(E_{F}\right)\right]$. We find, in agreement with tight-binding calculations, that Se contacts provide a larger conductance than $\mathrm{S}$ contacts (even though by only a factor of 3), and also that the conductance reaches a value close to the quantum of conductance with Te. The conductance thus increases with increasing atomic number. In the case of $\mathrm{S}$ and Se terminations, the Fermi levels (we recall that there is $0.01 \mathrm{eV}$ of difference in energy between the right and left Fermi levels) fall in the highest occupiedlowest unoccupied molecular-orbital (HOMO-LUMO) gap, while in the case of Te the HOMO is nearly exactly at the position of the Fermi levels. In the case of S, transport occurs via the tails of the LUMO orbitals that intersect the Fermi levels, and the LUMO is closer to the Fermi levels than the HOMO (see Fig. 4). The latter finding is in agreement with the fact that there is a charge transfer of about $0.1 e$ from the electrodes to the molecule, ${ }^{34}$ in practice providing a natural " $n$ doping" to the molecular region. ${ }^{2,4}$ On the other hand, in the case of Se, the HOMO is very close to the Fermi levels and the charge transfer occurs from the molecule to the electrodes, giving a slight " $p$ doping" character to the molecular region. However, the conductance increases only by about a 


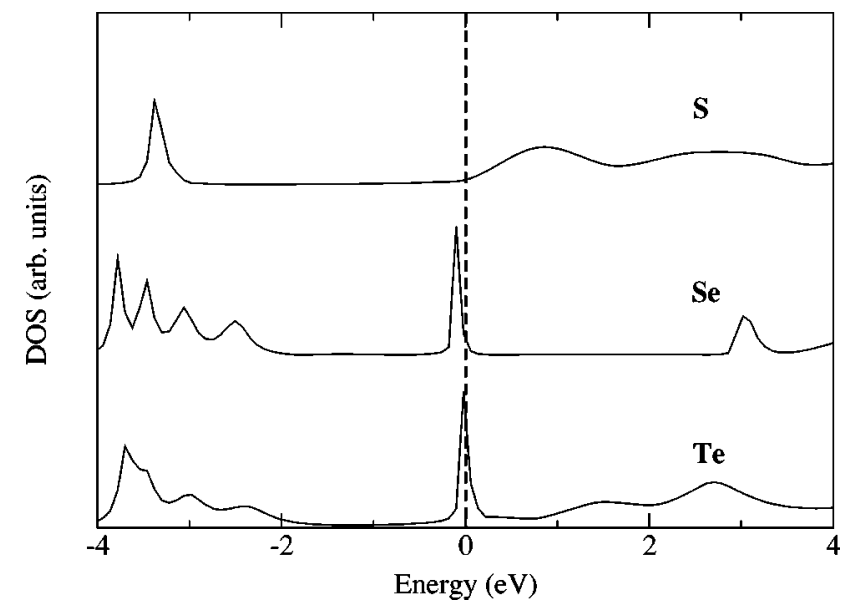

FIG. 4. Difference between the density of states of the two semi-infinite electrodes with and without the molecules of Fig. 3 in between. The left Fermi level has been chosen as the zero of energy. The right Fermi level is at $10 \mathrm{meV}$.

factor of 3, because the HOMO resonance is very narrow, and it is its tail that intersects the Fermi levels (see Fig. 4). These findings were recently confirmed experimentally. ${ }^{35} \mathrm{Fi}-$ nally, in the case of Te, an even larger charge transfer occurs from the molecular region to the electrodes (about $0.5 e$ ), pushing the HOMO practically into resonance with the left Fermi level. This transition from $n$ doping to $p$ doping of the molecular region is also consistent with the decrease of electronegativity of the end groups.

The conductance increase with increasing atomic number does not, however, correlate well with the increase in the density of states at the Fermi level. For instance, the ratio of the conductance $G$ for the $S$ - and Se-terminated molecules is $G(\mathrm{Se}) / G(\mathrm{~S})=3.3$, while the ratio of the respective DOS's at the Fermi level is 7.1. In the case of Te end groups, $G(\mathrm{Te}) / G(\mathrm{Se})=7.3$, and the DOS's ratio is 10.9 . However, we note that the contribution to the conductance of a given resonant mode is reduced by a factor approximately proportional to the ratio $\operatorname{DOS}\left(E_{F}\right) / \operatorname{DOS}\left(E_{\text {peak }}\right)$, where $\operatorname{DOS}\left(E_{\text {peak }}\right)$ is the peak value of the line shape of the corresponding resonance. ${ }^{36}$ Therefore, a better estimate of the conductance is provided by $G_{0} \times \operatorname{DOS}\left(E_{F}\right) / \operatorname{DOS}\left(E_{\text {peak }}\right)$ (taking the conductance at the peak to be unity). This quantity is reported in the last column of Table I. The agreement between the crude estimate provided by the above argument and the actual conductance is quite remarkable.

\section{CONCLUSIONS}

We have described a first-principles atomistic approach to calculate the electronic and atomic dynamics of nanoscale conductors under steady-state current flow, based on the selfconsistent solution of the Lippmann-Schwinger equation within the density-functional formalism. With the selfconsistent wave functions and relaxed ionic distributions obtained in the presence of current flow, any other transport quantity can, in principle, be calculated. We have applied the above approach to the case of transport in molecular wires with different anchoring groups at the interface between the molecule and the electrodes. In particular, we have found that conductances close to the quantum unit $\left(2 e^{2} / h\right)$ can be achieved with a given molecular structure, simply by increasing the atomic number of the anchoring group.

\section{ACKNOWLEDGMENTS}

We thank S.T. Pantelides for useful discussions, and for having collaborated with one of us (M.D.) on the problem of current-induced forces. One of us (M.D.) acknowledges support from the National Science Foundation Grant No. DMR01-02277, Carilion Biomedical Institute, and Oak Ridge Associated Universities. The calculations reported in this paper were performed on the SUN cluster of Virginia Tech's Computing Center.

\section{APPENDIX}

Here we give some details on the plane-wave representation of the different quantities presented above. For simplicity, we will assume that the sample is enclosed in a cubic box of side $2 L$. An extension to noncubic boxes is straightforward. The wave functions are expressed within the box as

$$
\Psi_{E \mathbf{K}_{\|}}(\mathbf{r})=\sum_{n} \Psi_{E \mathbf{K}_{\|}} e^{i \mathbf{k}_{\mathbf{n}} \cdot \mathbf{r}}
$$

with $\mathbf{n}=\left(n_{x}, n_{y}, n_{z}\right)$ an integer vector, and $\mathbf{k}_{\mathbf{n}}=\pi \mathbf{n} / L$. An equivalent expression holds for $\Psi^{0}$. The scattering potential $V[$ Eq. (4)] is

$$
V\left(\mathbf{r}, \mathbf{r}^{\prime}\right)=\sum_{n, n^{\prime}} e^{i \mathbf{k}_{\mathbf{n}} \cdot \mathbf{r}} V_{n n^{\prime}} e^{-i \mathbf{k}_{\mathbf{n}^{\prime}} \cdot \mathbf{r}},
$$

and the Lippmann-Schwinger equation (5) becomes

$$
\sum_{n^{\prime}} C_{E n n^{\prime}} \Psi_{E \mathbf{K}_{\|} n^{\prime}}=\Psi_{E \mathbf{K}_{\|} n}^{0},
$$

with

$$
C_{E n n^{\prime}}=\delta_{n n^{\prime}}-(2 L)^{6} \sum_{n^{\prime \prime}} G_{E n n^{\prime \prime}}^{0} V_{n^{\prime \prime} n^{\prime}},
$$

where $\delta_{n n^{\prime}}$ is the Kronecker delta. The Green's function in this representation is

$$
\begin{aligned}
G_{E n n^{\prime}}^{0}= & \frac{(-1)^{n_{x}+n_{y}+n_{x}^{\prime}+n_{y}^{\prime}}}{2 \pi^{2} L^{4}} \int_{0}^{\infty} t d t g_{E n_{z} n_{z}^{\prime}}(t / L) \\
& \times \int_{0}^{\pi / 2} d \phi \sin ^{2}(t \cos \phi) \sin ^{2}(t \sin \phi) \\
& \times\left(t^{2} \cos ^{2} \phi+\pi^{2} n_{x} n_{x}^{\prime}\right)\left(t^{2} \sin ^{2} \phi+\pi^{2} n_{y} n_{y}^{\prime}\right) \\
& \times\left[\left(t^{2} \cos ^{2} \phi-\pi^{2} n_{x}^{2}\right)\left(t^{2} \cos ^{2} \phi-\pi^{2} n_{x}^{\prime 2}\right)\right. \\
& \left.\times\left(t^{2} \sin ^{2} \phi-\pi^{2} n_{y}^{2}\right)\left(t^{2} \sin ^{2} \phi-\pi^{2} n_{y}^{\prime 2}\right)\right]^{-1}
\end{aligned}
$$

with 


$$
\begin{aligned}
\left.g_{E n_{z^{\prime} z} n^{\prime}}\left|\mathbf{K}_{\|}\right|\right)= & \frac{1}{W_{E \mathbf{K}_{\|}}} \int_{-L}^{L} d z e^{-i k_{n_{z}} z} \\
& \times\left[u_{E \mathbf{K}_{\|}}^{R}(z) \int_{-L}^{z} d z^{\prime} u_{E \mathbf{K}_{\|}}^{L}\left(z^{\prime}\right) e^{i k_{n_{z}^{\prime}} z^{\prime}}\right. \\
& \left.+u_{E \mathbf{K}_{\|}}^{L}(z) \int_{z}^{L} d z^{\prime} u_{E \mathbf{K}_{\|}}^{R}\left(z^{\prime}\right) e^{i k_{n_{z}^{\prime}} z^{\prime}}\right] .
\end{aligned}
$$

The functions $u_{E \mathbf{K}_{\|}}^{\alpha}(z)$, with $\alpha=L, R$, satisfy the equation

$$
\left[E+\frac{1}{2} \frac{d^{2}}{d z^{2}}-\frac{1}{2}\left|\mathbf{K}_{\|}\right|^{2}-v_{\text {eff }}(z)\right] u_{E \mathbf{K}_{\|}}^{\alpha}(z)=0,
$$

with left-moving (right-moving) boundary conditions for $\alpha$ $=L(\alpha=R)$. The quantity $W_{E \mathbf{K}_{\|}}$is the Wronskian,

$$
W_{E \mathbf{K}_{\|}}=u_{E \mathbf{K}_{\|}}^{L}(z) \frac{d}{d z} u_{E \mathbf{K}_{\|}}^{R}(z)-u_{E \mathbf{K}_{\|}}^{R}(z) \frac{d}{d z} u_{E \mathbf{K}_{\|}}^{L}(z),
$$

which is independent of $\mathrm{z}$.

The direct force on the ions, derived only from the bareelectrode wave functions $\Psi^{0}$, can be calculated with essentially arbitrary accuracy. The remaining force in Eq. (12) from the continuum wave functions is

$$
\begin{aligned}
\mathbf{F}= & -i(2 L)^{3} \int d E \int d^{2} \mathbf{K}_{\|} \sum_{n n^{\prime}}\left(\mathbf{k}_{\mathbf{n}}-\mathbf{k}_{\mathbf{n}^{\prime}}\right) \\
& \times e^{i\left(\mathbf{k}_{\mathbf{n}}-\mathbf{k}_{\mathbf{n}^{\prime}}\right) \cdot \mathbf{R}_{0}} V_{n n^{\prime}}^{p s}\left\{\Psi_{E \mathbf{K}_{\|} \Delta n}^{0} \delta \Psi_{E \mathbf{K}_{\|} \Delta n^{\prime}}^{*}\right. \\
& \left.+\delta \Psi_{E \mathbf{K}_{\|} \Delta n}\left[\Psi_{E \mathbf{K}_{\|} \Delta n^{\prime}}^{0}\right]^{*}+\delta \Psi_{E \mathbf{K}_{\|} \Delta n} \delta \Psi_{E \mathbf{K}_{\|} \Delta n^{\prime}}^{*}\right\},
\end{aligned}
$$

where $\delta \Psi_{E \mathbf{K}_{\|} \Delta n}=\Psi_{E \mathbf{K}_{\|} \Delta n}-\Psi_{E \mathbf{K}_{\|} \Delta n}^{0}$ and $\Psi_{E \mathbf{K}_{\|} \Delta n}, \Psi_{E \mathbf{K}_{\|} \Delta n}^{0}$ have been constructed according to Eq. (10), and the limit in Eq. (12) is understood. The term $V_{n n^{\prime}}^{p s}$ is the plane-wave representation of the nonlocal pseudpotential $v_{p s}\left(\mathbf{r}, \mathbf{r}^{\prime}\right)$.
${ }^{1}$ See, e.g., Molecular Electronics: Science and Technology, edited by A. Aviram and M.A. Ratner (New York Academy of Sciences, New York), 1998.

${ }^{2}$ M. Di Ventra, S. T. Pantelides, and N. D. Lang, Phys. Rev. Lett. 84, 979 (2000).

${ }^{3}$ M. Di Ventra, S.-G. Kim, S. T. Pantelides, and N. D. Lang, Phys. Rev. Lett. 86, 288 (2001).

${ }^{4}$ M. Di Ventra, S. T. Pantelides, and N. D. Lang, Appl. Phys. Lett. 76, 3448 (2000).

${ }^{5}$ N. D. Lang, Phys. Rev. B 52, 5335 (1995); 49, 2067 (1994); 45, 13599 (1992); 51, 2029(E) (1995).

${ }^{6}$ M. Di Ventra and S. T. Pantelides, Phys. Rev. B 59, R5320 (2000)

${ }^{7}$ J. Taylor, H. Guo, and J. Wang, Phys. Rev. B 63, 245407 (2001).

${ }^{8}$ N. Kobayashi, M. Brandbyge, and M. Tsukada, Phys. Rev. B 62, 8430 (2000).

${ }^{9}$ Y. Xue, S. Datta, and M. A. Ratner, J. Chem. Phys. 115, 4292 (2001).

${ }^{10}$ See, for instance, H. Ohnishi, Y. Kondo, and K. Takayanagi, Nature (London) 395, 780 (1998); A. Yazdani, D. M. Eigler, and N. D. Lang, Science 272, 1921 (1996); V. Rodrigues and D. Ugarte, Phys. Rev. B 63, 073405 (2001); N. D. Lang and Ph. Avouris, Phys. Rev. Lett. 84, 358 (2000).

${ }^{11}$ R. M. Metzger et al., J. Am. Chem. Soc. 119, 10455 (1997); C. Zhou, M. R. Deshpande, M. A. Reed, L. Jones, and J. M. Tour, Appl. Phys. Lett. 71, 611 (1997); M. A. Reed, C. Zhou, C. J. Muller, T. P. Burgin, and J. M. Tour, Science 278, 252 (1997); S. Datta, W. Tian, S. Hong, R. Reifenberger, J. I. Henderson, and C. P. Kubiak, Phys. Rev. Lett. 79, 2530 (1997); J. K. Gimzewski and C. Joachim, Science 283, 1683 (1999); J. Chen, M. A. Reed, A. M. Rawlett, and J. M. Tour, ibid. 286, 1550 (1999); Z. J. Donhauser et al., ibid. 292, 2303 (2001); M. A. Reed, J. Chen, A. M. Rawlett, D. W. Price, and J. M. Tour, Appl. Phys. Lett. 78,
3735 (2001); N. D. Lang and Ph. Avouris, Phys. Rev. B 64, 125323 (2001).

${ }^{12}$ P. Hohenberg and W. Kohn, Phys. Rev. B 136, 864 (1964); W. Kohn and L. J. Sham, Phys. Rev. 140, A1133 (1965). The exchange-correlation functional used in this work is the one of D. M. Ceperley and B. J. Alder, Phys. Rev. Lett. 45, 566 (1980), as parametrized by J. P. Perdew and A. Zunger, Phys. Rev. B 23, 5048 (1981).

${ }^{13}$ M. Di Ventra (unpublished).

${ }^{14}$ H. Ness, S. A. Shevlin, and A. J. Fisher, Phys. Rev. B 63, 125422 (2001).

${ }^{15}$ H. Ness and A. J. Fisher, Phys. Rev. Lett. 83, 452 (1999).

${ }^{16}$ Note that in this energy region, for some values of the momentum parallel to the electrode surfaces $\mathbf{K}_{\|}$, the solutions are also phase-shifted sine waves deep in the left electrode, which decay exponentially toward the right (see Ref. 5).

${ }^{17}$ The wave functions of $H_{0}$ (and, consequently, of $H$ ) in this energy region are also labeled according to the direction of propagation. We omit this label in the following, and discuss only the case of waves incident from the right.

${ }^{18}$ A. Messiah, Quantum Mechanics (Dover, New York, 1999), p. 801. Note that this form of scattering boundary conditions is valid only if the total Hamiltonian does not contain potentials that decay as $1 / r$.

${ }^{19}$ Other paths to self-consistency could of course be used, in which some or all of these charge modifications are eliminated. Many of such other choices lead to less stable procedures, and are harder to implement because the electrostatic potential without the charge modifications mentioned is no longer constant deep in the electrodes. Therefore, the wave functions deep in the electrodes would no longer be plane waves at intermediate stages of the calculation.

${ }^{20}$ G. B. Bachelet, D. R. Hamann, and M. Schlüter, Phys. Rev. B 26, 
4199 (1982); N. Troullier and J. L. Martins, ibid. 43, 1993 (1991).

${ }^{21}$ P. Pulay, in Modern Theoretical Chemistry, edited by H. F. Schaefer (Plenum, New York, 1977), Vol. 4.

${ }^{22}$ R. W. Hockney, in Methods in Computational Physics, edited by B. Alder, S. Fernbach, and M. Rotenberg (Academic, New York, 1970), Vol. 9, p. 135; R. W. Hockney and J. W. Eastwood, Computer Simulation Using Particles (McGraw-Hill, New York, 1981), p. 212.

${ }^{23}$ Strictly speaking, the term electromigration corresponds to current-induced motion of defects in solids (see, e.g., Ref. 24), but we use it here, in general terms, as the motion of any atom in the sample due to electron flow.

${ }^{24}$ R. Landauer and J. W. F. Woo, Phys. Rev. B 10, 1266 (1974).

${ }^{25}$ A. K. Das and R. Peierls, J. Phys. C 8, 3348 (1975).

${ }^{26}$ L. J. Sham, Phys. Rev. B 12, 3142 (1975).

${ }^{27}$ R. S. Sorbello, Solid State Physics, edited by H. Ehrenreich and F. Spaepen (Academic Press, New York, 1997), Vol. 51, p. 159, and references therein.
${ }^{28}$ M. Di Ventra and S. T. Pantelides, Phys. Rev. B 61, 16207 (2000).

${ }^{29}$ M. Di Ventra, S. T. Pantelides, and N. D. Lang (unpublished).

${ }^{30}$ N. D. Lang, S. Holloway, and J. K. Nørskov, Surf. Sci. 150, 24 (1985).

${ }^{31}$ Y. Takata et al., Surf. Sci. 259, 266 (1991).

${ }^{32}$ S. N. Yaliraki, M. Kemp, and M. A. Ratner, J. Am. Chem. Soc. 121, 3428 (1999).

${ }^{33}$ J. M. Seminario, A. G. Zacarias, and J. M. Tour, J. Am. Chem. Soc. 121, 411 (1999).

${ }^{34}$ This charge transfer has been calculated by integrating in a box the electron density of the molecule-electrode system minus the electron density of the free molecule and the bare electrodes. The dimension of the box perpendicular to the electrodes is equal to the electrode separation, and in each of the other two directions is equal to about $7 \AA$.

${ }^{35}$ J.-P. Bourgoin (private communication).

${ }^{36}$ V. Kalmeyer and R. B. Laughlin, Phys. Rev. B 35, 9805 (1987). 